\title{
Anomalous Extensor Indicis Proprius Muscle
}

\author{
Chul Han Kim \\ Department of Plastic and Reconstructive Surgery, \\ Soonchunhyang University College of Medicine, Seoul, Korea \\ Correspondence: Chul Han Kim \\ Department of Plastic and Reconstructive Surgery, Soonchunhyang University \\ College of Medicine, 59 Daesagwan-ro, Yongsan-gu, Seoul 140-743, Korea \\ Tel: +82-2-709-9283, Fax: +82-2-796-3543 \\ E-mail:kchann@hanmail.net \\ This work was supported in part by the Soonchunhyang University Research Fund. \\ No potential conflict of interest relevant to this article was reported. \\ Received: 25 Aug 2012 • Revised: 10 Oct 2012 • Accepted: 16 0ct 2012 \\ pISSN: 2234-6163・ elSSN: 2234-6171 \\ http://dx.doi.org/10.5999/aps.2013.40.1.79 • Arch Plast Surg 2013;40:79-81 \\ Copyright (C) 2013 The Korean Society of Plastic and Reconstructive Surgeons \\ This is an Open Access article distributed under the terms of the Creative Commons \\ Attribution Non-Commercial License (http://creativecommons.org/licenses/by-nc/3.0/) \\ which permits unrestricted non-commercial use, distribution, and reproduction in any \\ medium, provided the original work is properly cited.
}

Anatomic variations in the musculotendinous structure of the hand and wrist are particularly important in repairing or reconstruction of hand injuries. We report here an extensor indicis proprius (EIP) tendon with an unusual appearance, which is associated with dorsal wrist pain.

A 30-year-old man who worked as a cook presented with a painful mass-like lesion on the dorsum of the right hand (Fig. 1). The pain had been aggravated recently by forceful movement of the wrist over the previous 2 months, especially during cooking. At first, it was thought to be a ganglion. However, because the aspiration test was negative, it was clinically considered to be tenosynovitis. Because conservative management failed to relieve his symptoms, a sonographic examination was performed (Fig. 2). Anomalous muscle was identified, and the patient underwent surgical treatment. 


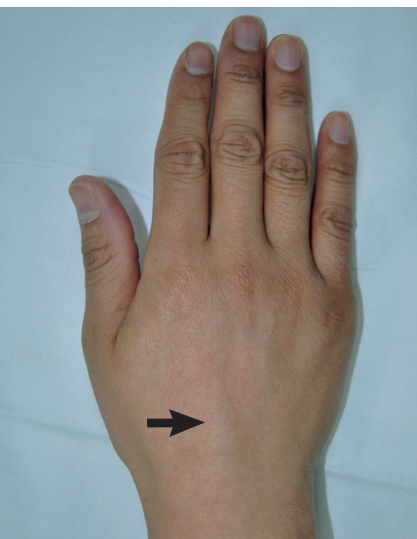

Fig. 1.

A 30-year-old man presented with pain on the dorsum of the right hand (black arrow).

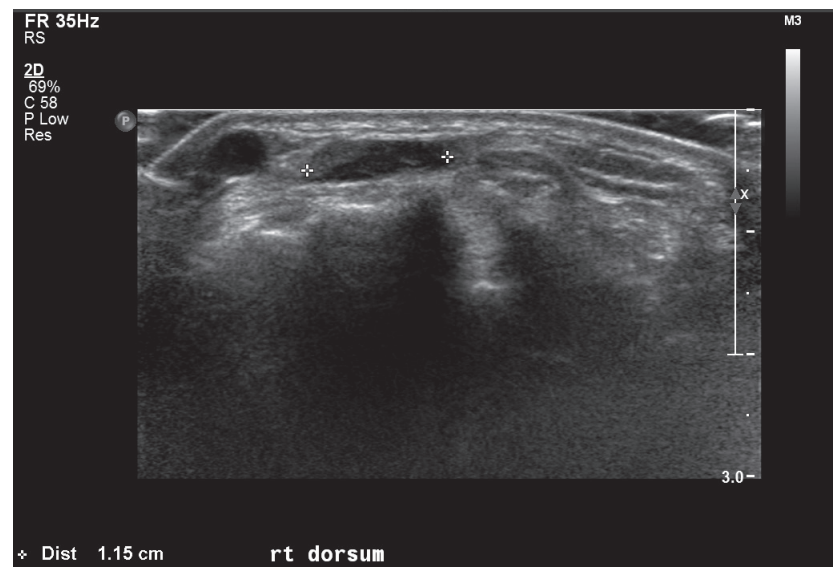

Fig. 2.

Ultrasonogram. The mass lesion revealed that an anomalous muscle (asterisks) had the typical echo texture of muscle tissue.
During surgery, there was found to be an abnormally situated muscle replacing the EIP tendon. Further dissection revealed an anomalous EIP muscle that passed along with the extensor digitorum communis (EDC) tendon to the extensor retinaculum of the fourth dorsal compartment and coursed to the index finger (Fig. 3). It was thought that the symptoms of the patient had resulted from the compression of the anomalous muscle by the extensor retinaculum of the fourth dorsal compartment. Therefore, the extensor retinaculum was released and the anomalous EIP muscle was partially excised (Fig. 4). No other pathologic findings such as a tenosynovitis or ganglion were found. The patient had an uneventful postoperative course and subsequently became asymptomatic and has done well since.

Anomalous extensor muscles of the hand that have been reported include anomalous EIP, extensor digitorum brevis manus (EDBM), extensor medii proprius (EMP), extensor indicis et medii communis (EIMC), and accessory belly of the dorsal interosseous muscle [1-5]. These anomalous extensor muscles have rarely been symptomatic. The EDBM, for example, was discovered by accident [1]. Because the proximal muscle belly of the EDBM is usually situated distal to the extensor retinaculum, it is less likely to produce symptoms. The EMP and EIMC are unlikely to cause symptoms because they have narrow width [2]. The accessory belly of the dorsal interosseous muscle usually inserts into the middle finger [2], and it is unlikely to be clinically found because it is very thin.

Anomalous EIP muscle has been reported previously $[2,3,5]$. Tan and Smith [2] reported that the anomalous EIP muscle belly extended beyond the fourth dorsal compartment, especially during full flexion of the wrist. Baker and Gonzalez [5] reported that the majority of the enlarged EIP muscle belly was situated beneath the extensor retinaculum. In addition, the EIP muscle was poorly developed, and this aberrant muscle was thus completely excised. Ritter and Inglis [3] described EIP syndrome, in which was found considerable tenosynovial proliferation surrounding the anomalous EIP muscle. However, the present case is different. The anomalous EIP muscle was sufficiently expanded distally beyond the extensor retinaculum without regard to wrist motion. Also, it functioned as a normal muscle and no other pathologic conditions were found intraoperatively. In our patient, the overlying mass-like lesion of the hand was caused by anomalous EIP muscle and the severe wrist pain during cooking probably resulted from muscle compression by the extensor retinaculum of the fourth dorsal compartment, subsequently leading to muscle ischemia and giving rise to ischemic pain.

The index finger has two independent tendons that are the least variable of the extensor tendons [2]. The EIP tendon is clinically significant, in that it is frequently used as a source for tendon transfer. In this case, the partial resection of the EIP muscle and decompression of the compartment with release of the tight extensor retinaculum were performed to allow free movement of the anomalous EIP muscle and normal extensor tendon. Total removal of the anomalous EIP muscle creates the risk of losing independent extension of the index finger. Thus, it was thought that decompression with partial muscle excision was the most 


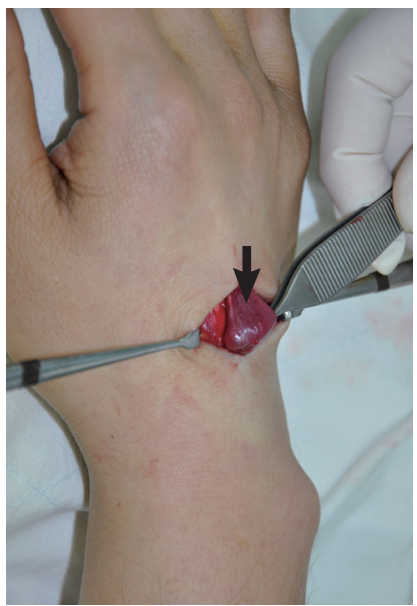

Fig. 3.

Intraoperative findings. The anomalous extensor indicis proprius muscle (black arrow) was identified expanding distally to the extensor retinaculum and running to the extensor hood of the index finger.

reasonable option.

In cases of anomalous EIP muscle, it may be difficult to clinically identify the anomalous muscle and distinguish it from a painful dorsal mass of the hand, if there is a lack of awareness of its existence. It may mimic a pathologic mass and is frequently misdiagnosed as a ganglion, tendon sheath cyst, tenosynovitis of the extensor tendons, exostosis, carpal boss, or benign soft-tissue tumor [1,2]. Ultrasonography may aid in diagnosis. On sonography of our case, the anomalous extensor muscle was identified along the ulnar aspect of the extensor tendon of the index finger showing a typical muscle-like echo texture.

In conclusion, even though anomalous EIP muscle is relatively rare and rarely causes dorsal wrist pain, awareness of this possible anatomic variation of the extensor tendon is important when considering tendon
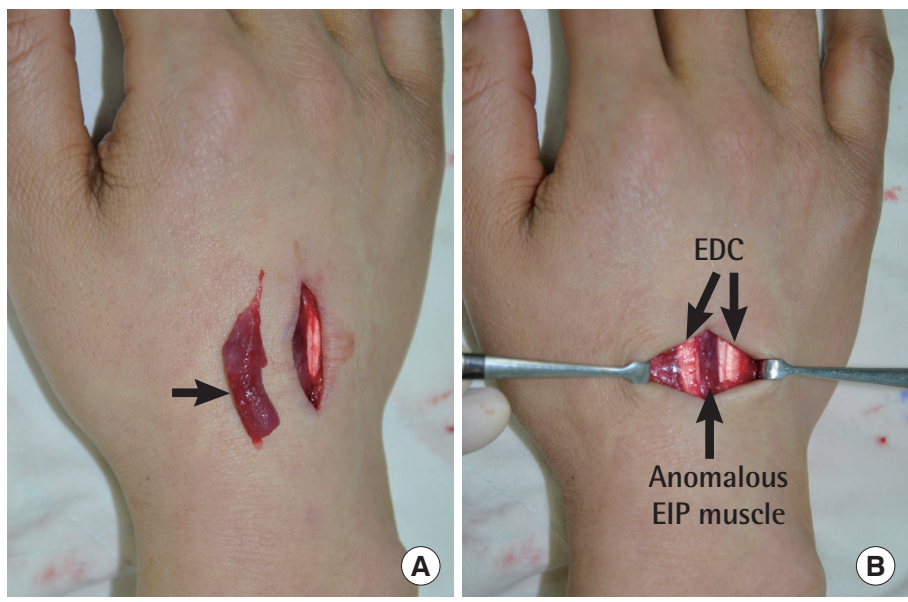

Fig. 4.

Intraoperative findings. (A) Excised anomalous extensor indicis proprius (EIP) muscle (black arrow). (B) After partial excision of the anomalous EIP muscle, the anomalous EIP muscle is shown in relationship to the extensor digitorum communis (EDC) tendons.

transfer or for managing hand disease.

\section{References}

1. Antonio A. Extensor digitorum brevis manus associated with a dorsal wrist ganglion: case report. Clin Anat 2008; 21:794-5.

2. Tan ST, Smith PJ. Anomalous extensor muscles of the hand: a review. J Hand Surg Am 1999;24:449-55.

3. Ritter MA, Inglis AE. The extensor indicis proprius syndrome. J Bone Joint Surg Am 1969;51:1645-8.

4. Still JM Jr, Kleinert HE. Anomalous muscles and nerve entrapment in the wrist and hand. Plast Reconstr Surg 1973;52:394-400.

5. Baker J, Gonzalez MH. Snapping wrist due to an anomalous extensor indicis proprius: a case report. Hand (NY) 2008;3:363-5. 